\title{
Research on low sub-surface damage detection of hard brittle optics materials
}

\author{
Dasen Wang ${ }^{1, a}$, Fengming Nie ${ }^{1, b}$, Ning Pei ${ }^{1, c}$, Guangping Zhang ${ }^{1, d}$, \\ ${ }^{1}$ Inner Mongolia institute of metal materials, Zhejiang,China.

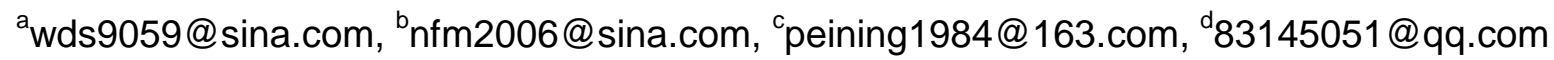

Keywords: optical; hard brittle material; detection;

Abstract. This paper is a low sub-surface damage detection method for hard and brittle optical materials. The method is based on the preparation of the test instrument and the detection of the surface damage of the sample to obtain the Brewster's angle of the sample to be tested and further utilize the Brewer The surface roughness of the sample is characterized by special angle. The method has the advantages of high accuracy and high detection method, and the detection method is simple and fast, and the surface of the sample to be tested is not damaged. This method can also be used to measure the other micro-surface structure of the optical element and to detect the surface microstructure .preparation of high quality high damage threshold of films. template explains and demonstrates how to prepare your camera-ready paper for Trans Tech Publications. The best is to read these instructions and follow the outline of this text.

\section{Introduction}

High quality hard brittle materials detection technology is the premise of high damage threshold of film, and higher damage threshold film has become one of the core issue of high power laser device, is restricted to develop in the direction of high energy, high power laser system of the main bottlenecks.

Hard and brittle optical materials in the manufacturing process of the surface and sub-surface defects in a certain energy under the laser irradiation, in the local high temperature, resulting in component damage. Therefore, it is of great practical significance and application prospect to study the sub-surface detection technology of hard and brittle optical materials. The scattered light of the measured optical element contains information mainly in two aspects. First, the intensity information can be described by the double reflection distribution function. Second, the polarization information can be described by the degree of polarization and retraction. Laser as a widely used high-quality light source, the light emitted is generally linearly polarized light, is the study of linear polarization reflection of the polarization state information is very important.

\section{Experimental}

(1) test equipment preparation

The test instrument comprises a dial, a stage, a laser, a first polarizer, a light intensity meter and a second polarizer, the dial being rotatable on a horizontal table, the upper surface edge of the dial having a circumference And said stage is arranged in the middle of the dial and is used to fix the sample to be measured.

The laser is arranged near the edge of the dial and the incident light emitted by the laser is just at the center of the dial and the stage, which is located between the laser and the dial and is used to filter the incident light Non - linearly polarized light.

(2) detection of sample surface damage

Adjust the object stage to level, will flake samples under test three boric acid lithium $\left(\mathrm{Li} \mathrm{B}_{3} \mathrm{O}_{5}\right)$ crystal vertical fixed in the middle of the object stage, open the laser and the light intensity meter, the laser power regulation to $5 \mathrm{w}$, turn the dial turns on the slide sample, adjusting the incident Angle, the process, keep the incoming light from the laser's axis line in the center of the object stage, the first 
polarizing film and perpendicular to the direction of incident light second polaroid perpendicular to the direction of the reflected light; Light intensity meter records each position in the process of the reflected light intensity, and at the same time record the position corresponding to the Angle of incidence, among them, the intensity of the weak position of the Angle of incidence is the brewster Angle; In this case, the intensity of light intensity is considered to be weakest when the intensity of light intensity is within the range of $0 \sim 300$ lumen/min. This implementation case measured three boric acid lithium $\left(\mathrm{Li} \mathrm{B}_{3} \mathrm{O}_{5}\right)$ crystals brewster Angle is $45^{\circ}$.

(3) Characterized the surface roughness of the sample using the measured Brewster angle.

According to the detection method of the present invention, when the incident angle is a specific angle, the reflected light is linearly polarized light, and its vibration direction is perpendicular to the incident surface. This specific angle is called the Brewster angle and is denoted by $\theta_{\mathrm{b}}$.

The following formula is the Brewster's law, where $n 1$ and $n 2$ are the refractive indices of the two kinds of media.

$$
\theta_{b}=\arctan \left(\frac{n_{2}}{n_{1}}\right)
$$

In the calculation of polarization theory, the Debye-Wakker factor is introduced to describe the interface roughness, ie

$$
\mathrm{DW}=\exp \left[-2\left(\frac{2 \pi \text { añcos } \theta_{\mathrm{b}}}{\lambda}\right)^{2}\right]
$$

Where, $\sigma$ is the surface roughness of the material, $\tilde{n}$ is the complex refractive index of the material, $\theta_{\mathrm{b}}$ is the positive incidence angle, $\lambda$ is the wavelength of the incident light, the non-ideal surface interface, Debye-Wakker factor approaches, $\mathrm{Rj}$ is the material of the reflected light intensity, ie

$$
R_{j} D W=R_{j}
$$

Thus, the incident angle is equal to the Brewster angle, which can represent the interface roughness. the surface roughness of the sample is represented by the measured brewster Angle, and the $\mathrm{Ra}$ is approximately $1.25 \mathrm{~nm}$.

The first polarizing plate is arranged in a direction perpendicular to the incident light, and the second polarizing plate is arranged in the direction of the vertically reflecting light. The use of lasers in the laboratory for the detection of surface damage is often used as a light source. Gas lasers often require no slight loss in the optical path of the output beam, which requires the use of Brewster windows and $\mathrm{Rp}=0$ but $\mathrm{tp}=1$ ( $\mathrm{Rp}$ is the P-polarized incident light, tp is the P-polarized transmitted light), that is, the P-polarized component is irradiated onto the Brewster window with a transmittance of $100 \%$ without loss. The reflection and transmission of the natural light incident on the surface of a dielectric material through a portion of the s-polarized light is reflected by the P-polarized light and the remaining S-polarized light. To obtain purely polarized polarized light. Considering that natural light has both reflection and refraction on the interface of the medium, it can be decomposed into two parts: one part is the $\mathrm{P}$ wave of the parallel and incident plane of the light vector; the other part is the $\mathrm{S}$ wave whose light vector is perpendicular to the incident plane. Thus, the reflected light and the refracted light generally become polarized light. When the incident angle of the incident light is equal to the Brewster angle, the reflected light becomes linearly polarized light. According to this principle, the polarizing plate can be used to obtain linearly polarized light. 


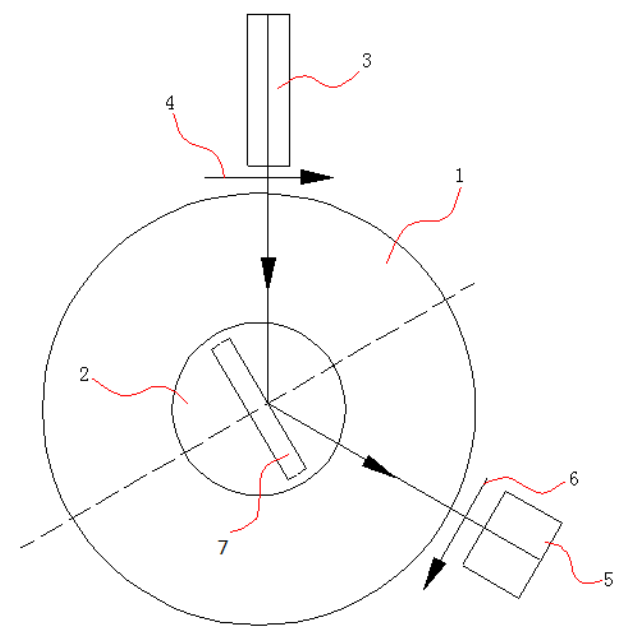

1- Scale, 2-platform, 3- laser, 4-first polaroid, 5- light intensity meter ;

6- second polaroid, 7-sample.

Fig 1: The light path diagram

In the above scenarios, the power of the described laser is $3 \sim 5 \mathrm{~W}$. When the light intensity data recorded in the light intensity meter is within the range of $0 \sim 300$ lumen/min, the light intensity is considered to be the weakest.

To ensure measurement accuracy, in the step (2) in the process of turning the dial, always keep the incoming light from the laser's axis line in the center of the object stage.

\section{Conclusions}

The structure of the test instrument is simple, the linearly polarized light is used to accurately measure the Brewster angle of the light incident on the sample, and the surface roughness of the sample can be characterized by keeping the measurement deviation within $1 / 100$, The detection method has the advantages of high accuracy and the detection method is simple and fast, and the surface of the sample to be tested is not damaged. Meanwhile, the method of the invention can also be used to measure other micro-surface structures of the optical element and to achieve the surface microstructure, Which provides the conditions for the preparation of high quality and high damage threshold films.

\section{Acknowledgements}

This work was financially supported by the International cooperation project (2015DFA10360), National defense infrastructure research project (JCKY2016208A002), General assembly technology project (41423020111), Ningbo natural fund(201601HJ-B01286).

\section{References}

[1] B. C. Gao, C. O. Davis, Examples of Using Imaging Spectrometry for Remote Sensing of the Atmosphere, Land, and Ocean, Proc. SPIE, 1998, Vol. 3502:[11]

[2] J. H. Qiu, An Improved Model of Surface BRDF-Atmospheric Coupled Radiation, IEEE Transactions on Geoscience and Remote Sensing, 2001, 39(1):182-187

[3] G.Franceschetti, A.Iodice, M.Migliaccio, and D.Riccio, Scatteing from natural rough surfaces modeled by fractional Browniam motion two-dimensional processes[J], IEEE Trans Antennas Propagat, 1999, Sept, Vol.47, pp.1405 1415

[4] Eric P. E. Lafortune , Sing-Choong Foo ,Kenneth E. Torrance and Donald P. Greenberg. "Non-linear approximation of reflectance function", Computer Graphics SIGGRAPH'97 Conference Proceedings), pp:117-126, August 1997. 